\title{
Belgeo
}

Revue belge de géographie

\section{Géographie des artistes dans les régions urbaines en Belgique}

The geography of artists in Belgium's urban areas

\section{Tatiana Debroux}

\section{(2) OpenEdition}

\section{Journals}

Édition électronique

URL : http://journals.openedition.org/belgeo/7913

DOI : $10.4000 /$ belgeo.7913

ISSN : 2294-9135

Éditeur :

National Committee of Geography of Belgium, Société Royale Belge de Géographie

\section{Édition imprimée}

Date de publication : 30 juin 2009

Pagination : 119-136

ISSN : 1377-2368

\section{Référence électronique}

Tatiana Debroux, «Géographie des artistes dans les régions urbaines en Belgique », Belgeo [En ligne],

2 | 2009, mis en ligne le 21 mai 2013, consulté le 01 mai 2019. URL : http://journals.openedition.org/ belgeo/7913 ; DOI : 10.4000/belgeo.7913

Ce document a été généré automatiquement le 1 mai 2019.

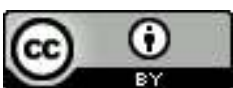

Belgeo est mis à disposition selon les termes de la licence Creative Commons Attribution 4.0 International. 


\section{Géographie des artistes dans les régions urbaines en Belgique}

The geography of artists in Belgium's urban areas

Tatiana Debroux

\section{Les artistes dans la littérature géographique : une problématique récente}

1 L'intérêt des géographes pour les artistes et les activités culturelles est apparu récemment : ces nouvelles thématiques de recherche se sont greffées aux problématiques urbaines et particulièrement aux questions des transformations sociales, urbanistiques et économiques des villes durant les trente dernières années $d u 20^{\circ}$ siècle. Les "stratégies culturelles" mises en place dans de nombreuses villes de par le monde pour relancer le dynamisme économique urbain ont notamment intéressé les chercheurs, soucieux de comprendre ces nouveaux développements et les formes qu'ils ont prises. A travers une gestion urbaine entrepreneuriale et une collaboration plus ou moins étendue avec des acteurs privés, les autorités locales ont investi dans la construction d'infrastructures culturelles et l'organisation de grands évènements dans l'espoir d'attirer investisseurs, consommateurs et nouveaux résidents aisés et ainsi encourager la dynamique économique urbaine dans son ensemble (Miles \& Paddison, 2005).

Outre le fait qu'il s'agit d'opérations relativement peu onéreuses par rapport à d'autres types d'investissements en infrastructures et qu'elles suscitent généralement l'adhésion de la population, le succès de telles entreprises s'explique également par la diffusion de nouvelles théories économiques inspirées de celles du capital humain, auxquelles appartiennent les travaux de Florida sur la "classe créative" (Florida, 2002; Shearmur, 2005). Ces théories font des infrastructures culturelles, des artistes et des différentes scènes urbaines des éléments-clés pour relancer la croissance économique, en attirant les travailleurs qualifiés qui composent la main-d'oeuvre d'entreprises des services 
spécialisés ou actives dans les secteurs des technologies, de l'information et des communications (Lloyd, 2002).

3 Si l'existence d'un lien entre le secteur culturel et le développement économique des villes est une vraie question, d'autant qu'il s'agit d'un secteur en croissance (Scott, 1997), les indicateurs et les corrélations développés par Florida ne peuvent suffire à démontrer un tel lien ni son sens (pour une discussion critique des travaux de Florida, voir notamment Levine, 2004 ; Glaeser, 2005 ; Peck, 2005 ; ou encore Vivant, 2006). Il importe donc de prendre du recul par rapport à ces assertions et de considérer plus en profondeur les rapports entre culture - envisagée ici en termes de création artistique - et développement urbain.

Dans un article récent, Markusen remet en cause la légitimité de la "classe créative" et montre notamment, à travers l'étude particulière des artistes, que les différents groupes professionnels rassemblés au sein de cette "classe" ont des comportements différents, entre autres sur le plan spatial (Markusen, 2006). L'auteure considère qu'il importe de distinguer les artistes, dont la géographie et le rôle dans l'économie urbaine sont bien plus complexes que ce que Florida n'avance. Pour estimer ce rôle, Markusen fait appel à la notion de "dividende artistique", "the additional economic impact that would not occur without the presence of artists", dont elle estime l'importance sur l'économie urbaine à travers la répartition des artistes dans les villes américaines (Markusen \& Schrock, 2006, p.1662). Une telle méthode avait déjà été employée auparavant par d'autres chercheurs désirant approcher l'activité artistique à travers la répartition spatiale des créateurs et interprètes, avec des résultats similaires sur le plan de la différenciation spatiale des territoires (Heilbrun, 1992; Blau et al., 1985). Ces travaux américains montrent que la géographie des artistes est un apport important et pertinent sur lequel une analyse des rapports entre activités culturelles et dynamiques urbaines contemporaines peut être bâtie.

5 En présentant la répartition spatiale des artistes en Belgique et en comparant sur ce point les régions urbaines du pays, cette étude prend place dans la vaste problématique des liens entre culture et développement urbain. Toutefois, il ne s'agit ici que de dresser un premier aperçu de la présence artistique en Belgique, sans pouvoir envisager à ce stade les conséquences économiques multiples pouvant résulter de la répartition observée.

6 L'accent est mis sur les personnes exerçant une profession dans le domaine artistique en tant que créateurs ou interprètes ${ }^{1}$; l'exercice de cartographie des artistes professionnels, de par les particularités de l'activité artistique, nécessite tout d'abord de définir la population étudiée sur le plan théorique et pratique, en fonction des sources disponibles.

7 A la lumière des observations réalisées dans les villes américaines, il a semblé important de ne pas comparer uniquement des nombres ou des proportions d'artistes entre agglomérations mais d'envisager également d'autres dimensions d'analyse.

Trois dimensions marquent donc l'examen de la répartition des artistes en Belgique : en premier lieu, pour distinguer finement les concentrations, plusieurs échelles d'analyse spatiale sont envisagées, permettant de comparer les villes entre elles mais également en leur sein. Deuxièmement, il est intéressant de différencier les professions regroupées et d'envisager la discipline artistique comme facteur de différenciation au niveau de la localisation résidentielle des artistes. En dernier lieu, il convient aussi d'introduire dans l'analyse la dimension historique, qui permet de se rendre compte que la géographie actuelle des artistes est le fruit de l'histoire récente - et ancienne - des régions urbaines. 
Ces trois dimensions permettent de dresser une esquisse du secteur artistique belge et de mieux appréhender les particularités et différences existant sur ce point entre les territoires. Elles montrent l'intérêt qu'il y a à dépasser l'approche simpliste des classements des villes selon le nombre d'artistes (ainsi que l'a fait Florida), pour envisager la diversité des situations et permettre ainsi d'enrichir les débats portant sur la présence artistique, la dynamique et les "performances" économiques des régions urbaines.

\section{Problèmes de définition théorique et pratique à l'étude de la population artistique}

Dans le langage courant, l'utilisation et les définitions du mot "artiste" sont nombreuses, désignant tour à tour les seuls peintres, les plasticiens ou tous les praticiens des beauxarts, tant créateurs qu'interprètes. La première étape pour tout chercheur s'intéressant aux artistes consiste donc à établir une définition du groupe, en fonction de ses objectifs : s'intéresse-t-il à une forme d'art ou plusieurs, aux métiers de la création ou de l'interprétation, aux amateurs ou aux professionnels? "Il n'existe pas en effet de critère univoque pour dire qui est artiste. (...) Pour des raisons matérielles (...) et idéologiques (...) l'identité professionnelle ne peut dépendre ni de l'exercice à plein temps de l'activité artistique, ni des ressources provenant de cette activité" (Moulin, 1983, p. 398). Le choix de critères s'impose pour la délimitation du champ artistique.

11 Contrairement à la plupart des autres catégories professionnelles dont l'appartenance est déterminée par des compétences particulières, la possession d'un diplôme et éventuellement l'affiliation à une association professionnelle, la catégorie des artistes est très hétérogène et soumise à des modifications fréquentes du fait de l'émergence de nouvelles pratiques artistiques. Ce groupe se caractérise également par de grands écarts de revenus, de durées de travail et par la pratique d'activités complémentaires, ce qui complique la délimitation des artistes "professionnels".

Pour ce faire et $d u$ fait du caractère souvent inapproprié des données statistiques existantes, certains chercheurs constituent leur propre échantillon en procédant par enquête, sur base de critères variés liés par exemple à la pratique artistique, la réception des oeuvres ou la reconnaissance de l'artiste (Karttunen, 1998).

D'autres préfèrent l'utilisation de données statistiques, dans un souci de plus grande exhaustivité : les choix effectués et la définition du groupe d'artistes dépendent alors du type de données accessibles au chercheur. Celles-ci, dont font partie les recensements, les enquêtes sur les forces de travail ou les données relatives à l'emploi, présentent souvent des problèmes de comparabilité et de définition des nomenclatures ${ }^{2}$, ainsi que des taux d'interrogation de la population et des temporalités de relevés variables. Effectuées dans plusieurs pays européens, ces constatations ont mené à l'instauration par le Comité du Programme Statistique de l'Union européenne d'un "leadership group" sur les statistiques culturelles (LEG-Culture) en 1997, dont la tâche était "d'établir, au niveau de l'UE, un système d'informations cohérentes et comparables susceptible de contribuer à une meilleure compréhension des liens entre la culture et le développement économique" (Commission européenne, 2000, p.2). Dans ce but ont été étudiés les instruments permettant la production d'indicateurs de l'emploi culturel, notamment l'utilisation de deux nomenclatures harmonisées, NACE et CITP-88 (codant respectivement les secteurs d'activité et les professions) et les limites en résultant. Ces deux nomenclatures - dans 
leur transposition belge - ont également été analysées dans une enquête commanditée par le Kunstenloket en vue de l'instauration d'un monitoring du secteur artistique en Communauté flamande (Loose \& Lamberts, 2006).

La définition des artistes professionnels dans le cadre de cet article se base sur le critère de la profession exercée et s'inspire des réflexions menées dans ces deux études. L'artiste professionnel est défini comme celui qui fournit des prestations artistiques ou produit des oeuvres contre rémunération en tant qu'interprète ou créateur, dans les secteurs de l'audiovisuel et des arts plastiques, de la musique, du spectacle, de la littérature, du théâtre, de la danse et de certains arts appliqués (le design et la mode). La nomenclature utilisée est la classification des professions utilisée par l'Institut National de Statistique pour le recensement belge de 1991.

L'emploi de cette édition du recensement s'explique par le fait qu'elle offre une nomenclature détaillée des professions et que l'ensemble de la population active a été interrogée sur ce critère, contrairement à l'édition de 2001 (dernière en date et rebaptisée Enquête socio-économique). Toutefois, dans la mesure où ce qui nous intéresse ici sont les structures de la géographie des artistes, les données de 1991 constituent une base solide pour une réflexion sur la situation contemporaine.

Il serait cependant erroné de penser atteindre l'exhaustivité parfaite dans le recensement des artistes selon cette méthode, pour les raisons précédemment évoquées liées aux particularités de l'activité artistique (absence ou mauvaise classification de certaines professions, pratique artistique à temps partiel et déclaration de l'activité principale ${ }^{3}$, etc.). Par ailleurs, à l'instar des données utilisées par les chercheurs américains, celles du recensement belge localisent les individus à leur lieu de résidence. En 1991, la population était également interrogée sur son lieu de travail (commune) : une comparaison a révélé de grandes similarités entre la localisation des artistes définie au lieu de résidence et au lieu de travail (coefficient de corrélation des deux répartitions de 0,91 ).

17 Prenant en compte ces remarques, le critère de la profession et les données du recensement de la population peuvent servir de base pour délimiter le groupe des artistes professionnels et étudier leur répartition géographique en Belgique.

\section{Etudier la répartition des artistes : échelles, disciplines artistiques et évolution temporelle}

18 Si l'importance des villes pour la création artistique et la concentration des artistes dans les grandes cités traversent toute l'histoire de l'art occidental, c'est parce que les espaces urbains ont toujours concentré les services et les publics, les cours et les mécènes dont les artistes ont besoin. Les créateurs et les interprètes y ont de ce fait toujours été présents en nombre et les centres-villes ont été les creusets de l'innovation artistique.

Les espaces urbains constituent donc l'échelle d'analyse privilégiée pour l'étude de la répartition spatiale des artistes ; classiquement, leur présence est appréhendée au niveau des aires métropolitaines à l'aide de données statistiques permettant une comparaison des territoires sur le plan du nombre d'artistes, envisagé de manière absolue et relative. Les observations menées par différents auteurs dans les villes américaines invitent cependant à dépasser cette simple approche et à considérer d'autres dimensions pour une meilleure compréhension de la présence artistique et de la différenciation des territoires selon ce critère. 
20 En premier lieu, il apparaît que cette population s'intéresse plus à certains espaces de la ville et particulièrement aux espaces centraux (offrant des avantages en matière d'accessibilité, de sociabilité et une typologie urbanistique variée - Bain, 2003). Au-delà d'une comparaison interurbaine de la présence d'artistes, il semble aussi intéressant de différencier les espaces constitutifs des aires métropolitaines et de distinguer notamment les centres-villes.

21 Par ailleurs, la plupart des travaux consacrés aux artistes se concentrent en réalité sur une discipline donnée - la peinture ou la musique le plus souvent - et ceux qui traitent d'un ensemble plus étoffé de pratiques artistiques ne les différencient généralement pas. Il est probable pourtant que toutes les catégories d'artistes n'aient pas les mêmes préférences spatiales. Dans une étude visant à tester empiriquement les conclusions de la littérature à propos de la présence des artistes en ville, des chercheurs ont mis en évidence des relations entre la structure socio-économique des agglomérations et la présence d'artistes dans les grandes villes américaines (Blau et al., 1985). Il ressort notamment de leurs analyses que les praticiens des arts de la scène se localisent préférentiellement dans des villes qui leur offrent une grande diversité de spectateurs, susceptibles de constituer des publics larges et multiples (ce qu'a également relevé Heilbrun, 1992). On peut supposer que ce sont dans les plus grandes villes, et a fortiori dans leurs parties centrales, que se rencontrent les publics larges et variés que recherchent les praticiens des arts de la scène, et que ces derniers se situent donc préférentiellement dans ces types de lieux. Distinguer les professions artistiques peut aussi révéler le degré de diversité des professions représentées dans les villes ou la spécialisation artistique de certaines d'entre elles; des chercheurs ont ainsi mis en évidence une plus grande diversité des professions artistiques dans les grandes villes et des spécialisations de niche dans des villes moyennes au Canada et aux Etats-Unis (Schimpf \& Sereda, 2007 ; Markusen et al., 2004).

22 Finalement, une troisième dimension sera abordée pour comprendre les particularités artistiques des villes, celle de leur évolution temporelle. Une ville dynamique sur le plan artistique attirera de nouveaux artistes, soucieux de profiter des nombreuses opportunités offertes par des scènes culturelles développées et diverses. A l'inverse, la mauvaise santé artistique de certaines villes pourrait être mise en relation avec la diminution du nombre de ses créateurs et interprètes durant une période antérieure. Les travaux de Markusen et King prennent en compte cette dimension temporelle, en comparant la croissance du nombre d'artistes dans quelques métropoles américaines et l'évolution de la population active totale entre 1980 et 1990 (Markusen \& King, 2003). Bien que la mise en oeuvre de telles analyses temporelles pose souvent des difficultés au niveau de la comparabilité des données, elles apportent certainement une clef d'interprétation supplémentaire au positionnement respectif des villes sur le plan artistique à un moment donné, et doivent donc être tentées.

\section{Le cas des régions urbaines en Belgique}

Pour analyser la répartition des artistes professionnels en Belgique, les données individuelles du recensement de 1991 ont été regroupées au niveau communal. Les 18 régions urbaines qui sont considérées, délimitées dans Luyten \& Van Hecke (2007), se décomposent en un noyau urbain, éventuellement une couronne urbaine et une banlieue. Une modification a toutefois été apportée au découpage proposé pour la pertinence des 
comparaisons entre régions urbaines : elle consiste à étendre le noyau urbain de Bruxelles aux 19 communes de la Région bruxelloise.

\section{Répartition des artistes sur le territoire belge}

En Belgique, un peu plus de treize mille personnes déclaraient exercer à titre principal une profession artistique en Belgique en 1991. Parmi ces 13092 artistes, 9345 étaient domiciliés dans l'une des 18 régions urbaines belges; ce chiffre s'explique a priori par l'étendue de ces régions et leurs fortes densités de population. Toutes les régions urbaines sont-elles pour autant attractives pour les artistes et comment se répartissent-ils au sein de ces régions? La figure 1 permet de répondre à ces interrogations. Le nombre total d'artistes y est représenté pour chaque commune, à l'exception de la Région de BruxellesCapitale, pour laquelle les données des 19 communes sont regroupées, pour plus de lisibilité. Un encart présente le détail des communes bruxelloises.

Figure 1. Artistes au lieu de résidence (1991).

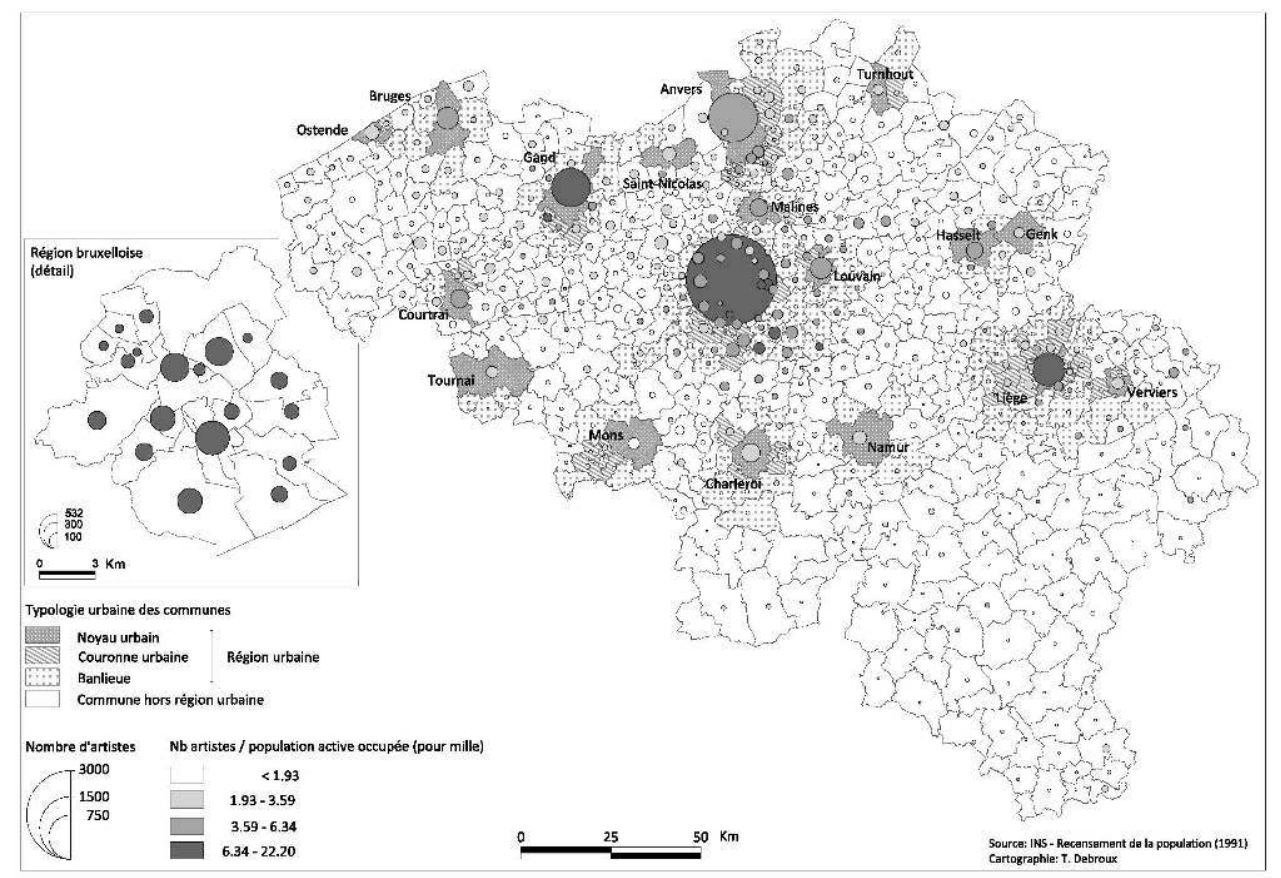

La première constatation est sans aucun doute la concentration des artistes dans les villes, puisque $71 \%$ d'entre eux résident dans une des 18 régions urbaines. La région urbaine bruxelloise abrite sans conteste la plus grande concentration ( $34 \%$ des artistes du pays), plus importante que ne le laisse prévoir la part de la population totale qui y vit (17\%). La part d'artistes dans les autres régions urbaines suit globalement un gradient décroissant en fonction de leur population totale, les villes wallonnes - excepté Liège comptant néanmoins moins d'artistes qu'attendu. Le mauvais classement en termes du nombre total d'artistes est interpellant pour Charleroi et Mons $^{4}$ : cependant, ceci peut partiellement résulter d'une différence dans la structure de leurs populations, ce qui ne peut se percevoir à travers la simple observation des nombres absolus d'artistes.

Les cercles de la figure 1, de surface proportionnelle au nombre total d'artistes professionnels, ont donc été tramés en fonction de la part que les artistes représentent 
dans la population active occupée de la commune. Ceci conforte la différence entre la Flandre et la Wallonie au niveau des artistes qui, hormis dans la périphérie bruxelloise et la région urbaine de Liège, sont proportionnellement moins représentés en Région wallonne, tant dans les régions urbaines que dans les communes n'en faisant pas partie ${ }^{5}$. Le tableau 1 synthétise cette information pour les régions urbaines. A cette échelle, Bruxelles est toujours dominante parmi les villes belges $(7,47 \%)$; cependant, Gand et Louvain ont dépassé Anvers, qui se retrouve en quatrième position, juste avant Liège $(4,14 \%$ \%). La dernière grande ville du pays, Charleroi, se retrouve avant-dernière avec un peu moins de deux artistes pour mille actifs occupés, une situation proche des autres régions urbaines wallonnes. 
Tableau 1. Part d'artistes dans la population active occupée (\%).

\begin{tabular}{|c|c|c|c|c|}
\hline Ville & Noyau urbain & Couronne urbaine & Banlieue & Région urbaine \\
\hline Bruxelles & 10,18 & 4,56 & 4,53 & 7,47 \\
\hline Anvers & 5,53 & 3,11 & 2,69 & 4,18 \\
\hline Bruges & 4,03 & & 2,44 & 3,56 \\
\hline Courtrai & 4,23 & 2,68 & 1,59 & 3,24 \\
\hline Gand & 6,55 & 4,57 & 2,46 & 4,93 \\
\hline Genk & 2,17 & & 2,12 & 2,15 \\
\hline Hasselt & 3,87 & & 3,46 & 3,69 \\
\hline Louvain & 5,18 & 3,90 & 3,58 & 4,47 \\
\hline Malines & 4,21 & 3,19 & 2,59 & 3,60 \\
\hline Ostende & 3,20 & 2,25 & 0,92 & 2,82 \\
\hline Saint-Nicolas & 2,71 & & 1,49 & 2,49 \\
\hline Turnhout & 2,68 & 2,29 & 1,87 & 2,37 \\
\hline Charleroi & 2,21 & 1,57 & 1,54 & 1,87 \\
\hline Liège & 7,49 & 2,78 & 2,97 & 4,14 \\
\hline Mons & 1,86 & 1,35 & 1,11 & 1,50 \\
\hline Namur & 2,04 & & 1,86 & 1,99 \\
\hline Tournai & 2,15 & & 1,91 & 2,10 \\
\hline Verviers & 3,17 & 2,25 & 2,71 & 2,85 \\
\hline Belgique $(3,59)$ & 6,14 & 3,35 & 2,99 & 4,70 \\
\hline
\end{tabular}

Source : INS - Recensement de la population, 1991

Les trois premières colonnes du tableau permettent par ailleurs de comparer les proportions d'artistes dans la population active occupée au sein des zones constituant les régions urbaines : dans tous les cas, c'est dans le noyau urbain que la part d'artistes est la plus élevée (particulièrement à Bruxelles, où les artistes sont proportionnellement deux fois plus nombreux à habiter au centre que dans le reste de la région urbaine). Cette part suit ensuite un gradient décroissant, depuis le noyau urbain jusque la banlieue, hormis dans le cas de Liège et Verviers. La population des artistes privilégie visiblement les régions urbaines et à l'intérieur de celles-ci, les communes centrales. 
Deux constatations principales sont à tirer : d'une part, la carte réalisée met en évidence le rôle de pôle d'attraction que constituent la capitale et les villes - particulièrement leurs parties centrales - sur les artistes. La deuxième constatation qui s'impose concerne la différence d'attractivité des deux grandes régions: la Wallonie abrite un moins grand nombre d'artistes mais surtout, ceux-ci représentent une proportion plus faible de la population active occupée qu'en région flamande (Liège constituant l'exception). Cette différence est interpellante, car elle se joue à tous les niveaux, tant urbain que non urbain. Le cas des régions urbaines est cependant particulièrement frappant en regard de la situation en Flandre, où même les plus petites villes ont une proportion d'artistes plus élevée que les villes wallonnes de plus grande taille: doit-on en conclure que celles-ci ne sont pas attractives globalement pour la population étudiée ou que le nombre réduit d'artistes y résulte d'une moins grande diversité des professions représentées? Une deuxième phase de l'analyse a alors consisté à différencier les artistes en fonction de la discipline qu'ils exercent, afin de porter un autre regard sur leur répartition, de mettre en évidence d'éventuelles spécificités locales au niveau des régions urbaines et d'ainsi nuancer les résultats obtenus.

\section{Différenciation des artistes selon les formes d'art exercées}

Les quelques 86 professions artistiques retenues dans la classification de l'INS ont été rassemblées en huit catégories: les plasticiens (au sens large, y compris les photographes), les designers et décorateurs, le secteur de la mode, la littérature, la danse, la musique, une catégorie regroupant les artistes du spectacle vivant (arts du cirque, variétés et music-hall) ainsi qu'une catégorie mixte - du fait de la nomenclature consacrée au théâtre et à l'audiovisuel (cinéma et télévision) ${ }^{6}$. Pour étudier les particularités des régions urbaines, un coefficient de spécificité des catégories a été mis en place: il rapporte l'effectif d'une catégorie au nombre total d'artistes dans un lieu donné à cette même proportion établie pour l'ensemble des territoires étudiés (ici, l'ensemble des régions urbaines). Ce rapport est supérieur à 1 dans le cas d'une catégorie plus spécifique dans une région que dans l'ensemble du territoire d'étude, et inférieur à 1 si cette catégorie y est proportionnellement moins représentée.

Le tableau 2 présente ces coefficients de spécificité pour les 18 régions urbaines. Par ailleurs, les mêmes coefficients ont été calculés en distinguant les trois zones constitutives des régions urbaines: le tableau 3 synthétise cette information, en indiquant dans quelles proportions les territoires où une catégorie est spécifique sont des noyaux urbains, des couronnes urbaines ou des banlieues.

Tableau 2. Coefficients de spécificité des disciplines artistiques (régions urbaines).

\begin{tabular}{|l|l|l|l|l|l|l|l|l|}
\hline $\begin{array}{l}\text { Région } \\
\text { urbaine }\end{array}$ & $\begin{array}{l}\text { plasticiens } \\
46 \%\end{array}$ & $\begin{array}{l}\text { déco/ } \\
\text { design } \\
15 \%\end{array}$ & $\begin{array}{l}\text { mode } \\
5 \%\end{array}$ & $\begin{array}{l}\text { littérature } \\
3 \%\end{array}$ & $\begin{array}{l}\text { spectacle } \\
\text { vivant } 1 \%\end{array}$ & $\begin{array}{l}\text { musique } \\
14 \%\end{array}$ & $\begin{array}{l}\text { danse } \\
2 \%\end{array}$ & $\begin{array}{l}\text { théâ/ } \\
\text { audiovis. } \\
14 \%\end{array}$ \\
\hline Bruxelles & 1,01 & $\mathbf{0 , 8 8}$ & $\mathbf{1 , 2 0}$ & 0,93 & $\mathbf{0 , 6 9}$ & $\mathbf{0 , 8 9}$ & $\mathbf{0 , 7 1}$ & $\mathbf{1 , 2 4}$ \\
\hline Anvers & $\mathbf{0 , 9 3}$ & $\mathbf{1 , 2 3}$ & 0,97 & $\mathbf{1 , 6 0}$ & $\mathbf{0 , 5 3}$ & $\mathbf{0 , 8 2}$ & $\mathbf{1 , 7 2}$ & 1,00 \\
\hline Bruges & 1,10 & $\mathbf{1 , 4 9}$ & 0,68 & 0,81 & 1,83 & 0,94 & 0,99 & $\mathbf{0 , 2 6}$ \\
\hline
\end{tabular}




\begin{tabular}{|c|c|c|c|c|c|c|c|c|}
\hline Courtrai & 1,16 & 1,49 & 1,99 & 0,39 & 0 & 0,59 & 1,43 & 0,16 \\
\hline Gand & 0,93 & 1,01 & 1,09 & 0,87 & 2,83 & 1,15 & 0,88 & 0,93 \\
\hline Genk & 1,34 & 1,48 & 0 & 0 & 1,45 & 0,60 & 0 & 0,38 \\
\hline Hasselt & 1,21 & 1,62 & 0,76 & 1,06 & 0,48 & 0,48 & 0,52 & 0,34 \\
\hline Louvain & 0,91 & 1,12 & 0,47 & 2,51 & 0 & 0,81 & 0,64 & 1,39 \\
\hline Malines & 0,80 & 0,94 & 0,25 & 1,70 & 5,29 & 1,31 & 0 & 1,31 \\
\hline Ostende & 1,02 & 1,52 & 0,24 & 0,82 & 0 & 1,15 & 2,49 & 0,32 \\
\hline $\begin{array}{l}\text { Saint- } \\
\text { Nicolas }\end{array}$ & 0,82 & 1,45 & 2,44 & 0,91 & 2,05 & 1,10 & 2,21 & 0,27 \\
\hline Turnhout & 1,40 & 1,07 & 1,96 & 1,64 & 1,24 & 0,20 & 0 & 0,11 \\
\hline Charleroi & 1,15 & 1,24 & 0,49 & 0,16 & 1,11 & 1,01 & 1,79 & 0,42 \\
\hline Liège & 0,96 & 0,62 & 0,40 & 0,54 & 1,02 & 1,98 & 1,54 & 0,72 \\
\hline Mons & 1,20 & 0,72 & 0 & 0 & 1,67 & 1,17 & 0,45 & 1,02 \\
\hline Namur & 1,13 & 1,04 & 1,10 & 1,11 & 1,67 & 1,17 & 0,45 & 0,29 \\
\hline Tournai & 1,20 & 1,01 & 1,02 & 0 & 1,30 & 0,54 & 2,09 & 0,79 \\
\hline Verviers & 1,17 & 1,00 & 0,48 & 0,40 & 0,92 & 1,44 & 0 & 0,40 \\
\hline
\end{tabular}

Note: Les coefficients de spécificité significatifs sont indiqués en gras.

Source : INS - Recensement de la population, 1991

31 La catégorie des plasticiens constitue sans nul doute le groupe le plus fourni (46\% de l'ensemble des artistes recensés). Elle n'est spécifique que dans des régions urbaines de petite taille (Turnhout puis Genk et Hasselt). La largeur de cette catégorie empêche l'apparition de réelles spécificités, et quand c'est le cas, le fait qu'elles se produisent dans des petites villes et dans les banlieues de celles-ci, laisse supposer qu'il s'agit plutôt de spécialisations par défaut.

32 Avec $15 \%$ des artistes, la catégorie consacrée aux professions du design et de la décoration est la deuxième en ordre d'importance. Elle est presque exclusivement spécifique aux villes flamandes, particulièrement celles de petite taille (Hasselt, Ostende, Courtrai et Bruges possèdent des coefficients de spécificité supérieurs ou égaux à 1,5 ). Outre les petites villes citées, cette catégorie est aussi spécifique - mais dans une moindre mesure - à Anvers. Un schéma semblable se dessine pour la catégorie relative aux métiers de la mode, plus souvent représentée dans des petites villes, en Flandre: nettement spécifique à Saint-Nicolas, Courtrai et Turnhout (coefficients de spécificité proches de 2), elle l'est cependant aussi à Bruxelles.

33 L'analyse des spécificités selon les types d'espaces montre que les catégories du design et de la mode sont plus souvent surreprésentées dans les espaces centraux, puis dans les 
banlieues des régions urbaines. Les spécialisations observées pour ces catégories dans des petites villes flamandes (avec Anvers et Bruxelles comme exceptions) sont sans doute à mettre en relation avec une certaine tradition manufacturière (meubles) et textile de plusieurs de ces régions urbaines. Par ailleurs, de par leur position en bordure du champ artistique et la nature des biens produits, les professionnels regroupés dans ces deux catégories ressentent probablement moins l'impératif d'une localisation centrale, à proximité des autres artistes, et trouvent sans doute d'autres avantages à ces localisations plus périphériques (p.ex., proximité avec d'autres secteurs d'activités).

Une toute autre logique de localisation apparaît dans le cas de la catégorie mixte consacrée au théâtre et à l'audiovisuel (14\% des artistes). Les coefficients de spécificité les plus élevés sont ceux de Louvain et Bruxelles. La différenciation des régions urbaines indique qu'il s'agit d'une catégorie plus fréquemment spécifique dans les noyaux urbains et plus rarement à mesure que l'on s'éloigne du centre (tableau 3). Capitale du pays, Bruxelles abrite une grande diversité de métiers dans ces secteurs et se distingue de Louvain par une présence forte de comédiens et d'acteurs (alors que ce sont les réalisateurs qui dominent dans la cité louvaniste). La présence marquée de comédiens et d'acteurs à Bruxelles, qui existe aussi - mais dans une moindre mesure - à Anvers, Liège et Gand, va dans le sens des conclusions de Blau et al. (1985) qui mettaient en évidence l'importance pour les artistes de la scène de se situer près de larges publics diversifiés, tels qu'on peut les rencontrer dans les plus grandes villes (et a fortiori dans leur centre).

Tableau 3. Spécificité des disciplines dans les zones constitutives des régions urbaines (\%).

\begin{tabular}{|l|l|l|l|l|l|l|l|l|}
\hline & plasticiens & $\begin{array}{l}\text { déco/ } \\
\text { design }\end{array}$ & mode & littérature & $\begin{array}{l}\text { spectacle } \\
\text { vivant }\end{array}$ & musique & danse & $\begin{array}{l}\text { théâ/ } \\
\text { audiovis. }\end{array}$ \\
\hline $\begin{array}{l}\text { Noyau } \\
\text { urbain }\end{array}$ & 30,30 & 40,74 & 54,55 & 60,00 & 50,00 & 52,94 & 77,78 & 58,33 \\
\hline $\begin{array}{l}\text { Couronne } \\
\text { urbaine }\end{array}$ & 27,27 & 22,22 & 18,18 & 10,00 & 16,67 & 23,53 & 22,22 & 25,00 \\
\hline Banlieue & 42,42 & 37,04 & 27,27 & 30,00 & 33,33 & 23,53 & 0,00 & 16,67 \\
\hline & 100,00 & 100,00 & 100,00 & 100,00 & 100,00 & 100,00 & 100,00 & 100,00 \\
\hline
\end{tabular}

Note : Ce tableau se lit « $30 \%$ des entités où la catégorie des plasticiens est spécifique correspondent à un noyau urbain ».

Source : INS - Recensement de la population, 1991

Représentée également à hauteur de $14 \%$ des artistes, la catégorie des musiciens comprend également bon nombre d'artistes de la scène: tout comme le groupe précédent, elle est le plus souvent spécifique dans les noyaux des régions urbaines. L'analyse du tableau 2 révèle une forte spécificité liégeoise dans ce domaine.

La surreprésentation de musiciens à Liège est principalement le fait d'artistes interprètes; il est intéressant de constater que cette région urbaine abrite toujours aujourd'hui le plus grand nombre de violonistes parmi les villes belges, proportionnellement au nombre total de musiciens. Ceci semble refléter une certaine pérennité dans la localisation de certains groupes d'artistes; au 19e siècle en effet, une 
école de violon s'est développée à Liège autour des personnalités d'Henri Vieuxtemps, puis d'Eugène Ysaye. De manière générale, la présence importante de musiciens classique à Liège est sans doute à rapprocher à la présence de l'Opéra Royal de Wallonie, de l'Orchestre Philharmonique et du Conservatoire Royal de Liège. Contrairement aux interprètes, les compositeurs ne sont par contre pas nombreux à Liège. En réalité, la plupart de ceux-ci se concentrent à Bruxelles et à Anvers, villes pour lesquelles la catégorie des musiciens n'est pas spécifique, mais qui n'en abritent pas moins les créateurs en matière musicale ${ }^{7}$.

La catégorie précédente a mis pour la première fois en évidence une spécificité forte en Wallonie; la danse constitue l'autre catégorie spécifique dans deux régions urbaines wallonnes. Les coefficients de spécificité de Charleroi et Liège sont toutefois comparables à celui d'Anvers $(1,7)$, et inférieur à celui d'Ostende $(2,5)^{8}$. L'analyse du tableau 3 met finalement en évidence le caractère central très marqué de cette catégorie, spécifique dans $77 \%$ des cas dans des noyaux urbains (et parmi ceux-ci, dans ceux des grandes villes). Les artistes de cette catégorie, tout comme les comédiens et les musiciens, font partie des artistes de la scène. Tout comme eux, ils se localisent préférentiellement là où existent de vastes publics diversifiés - particulièrement dans le cas de la danse contemporaine qui touche un public très spécialisé -, dans des villes de taille importante où se trouvent par ailleurs les lieux de formation. Ceux-ci tournent souvent autour de la personnalité d'un chorégraphe et il n'est donc pas étonnant de constater que Bruxelles et Anvers abritent presque l'entièreté des chorégraphes du royaume en 1991, alors que la spécificité de la danse dans des régions urbaines plus petites résulte de la présence d'interprètes.

Une autre catégorie est constituée d'artistes de la scène, celle du spectacle vivant (essentiellement des artistes de variété et de music-hall). Ne regroupant qu'un nombre très réduit de personnes ( $1 \%$ des artistes), cette catégorie n'est spécifique que dans les régions urbaines de Malines et Gand (particulièrement leur centre), de taille plus modeste que les villes où étaient spécifiques les catégories des arts de la scène précédemment évoquées.

La dernière catégorie, enfin, est constituée des professions artistiques littéraires. Cette catégorie est plus souvent spécifique dans les noyaux urbains que dans le reste des régions urbaines; l'analyse des spécificités pour l'ensemble de ces régions fait ressortir de manière significative Louvain et Anvers. Par contre, aucune région urbaine wallonne ne se démarque par la présence des artistes littéraires, souvent absents ou présents en très petits effectifs. Cette faiblesse du champ littéraire en Wallonie, interpellante en regard de la situation en Flandre, procède sans doute d'une concentration des auteurs francophones à Bruxelles, ou d'une moins grande présence d'écrivains en Belgique francophone résultant de l'attrait qu'exerce la France voisine sur ceux-ci.

La localisation des artistes (considérés sans distinction aucune) faisait ressortir la faiblesse de la scène artistique wallonne par rapport à celle de la Flandre; la distinction des disciplines artistiques révèle des comportements différenciés selon la nature des groupes. En Flandre, la spécialisation de villes petites et moyennes dans les secteurs de la mode et du design témoigne d'un pôle plus "marginal" des professions artistiques, pour lesquelles la centralité n'est pas une condition nécessaire à l'activité. A l'inverse, les formes d'art liées à la scène se concentrent de manière générale dans les espaces centraux des grandes agglomérations, où se trouvent de nombreux lieux de représentation et des publics variés. Une différence se marque cependant entre la 
catégorie des arts du spectacle plus populaires (spectacle vivant), spécifique dans des villes de taille modeste, et le théâtre, la musique et la danse, surreprésentés dans des villes plus importantes.

De manière générale, la fréquente sous-représentation des catégories de professions les plus fournies dans les villes wallonnes est à rapprocher des faibles effectifs d'artistes qui y vivent, relativement à leur population.

Enfin, au-delà de leurs particularités respectives (spécificité de la danse, de la littérature et du design à Anvers et de la mode et de la catégorie théâtre/audiovisuel à Bruxelles), les deux grandes régions urbaines du pays apparaissent comme celles qui abritent la plus grande diversité de professions artistiques et particulièrement les franges créatives de ces catégories (dans le cas de la musique et de la danse par exemple, les compositeurs et les chorégraphes y sont proportionnellement plus présents).

\section{Dynamique artistique des régions urbaines durant la décennie 1981-1991}

43 La prise en compte de l'évolution de la présence des artistes au sein des villes belges parachève ce tableau : les villes qui abritent le plus d'artistes en 1991 sont-elles celles qui les ont attirés lors de la décennie précédente? Comment les deux régions du pays se différencient-elles suivant ce critère?

Ce sont des données du recensement précédant celui de 1991 qui ont été utilisées pour cette analyse. La nomenclature des professions de 1981 étant beaucoup moins détaillée que celle de 1991, seuls deux types d'artistes ont pu être utilisés pour construire un indicateur d'évolution. Il s'agit, d'une part, des artistes plasticiens et d'autre part, des professions artistiques liées au design et à la décoration (les deux catégories les plus fournies en 1991). Le tableau 4 présente le résultat de ces comparaisons, rapporté à l'évolution de la population active occupée des régions urbaines au cours de la décennie 1980 (dernière colonne).

Tableau 4. Evolution de la proportion d'artistes dans la population active occupée (1981 - 1991).

\begin{tabular}{|l|l|l|l|l|l|l|l|}
\hline $\begin{array}{l}\text { Région } \\
\text { urbaine }\end{array}$ & $\begin{array}{l}\text { Nb } \\
\text { artistes } \\
\text { '81 A }\end{array}$ & $\begin{array}{l}\text { Nb } \\
\text { artistes } \\
\text { '91 B }\end{array}$ & $\begin{array}{l}\text { Evol. } \\
\text { artistes } \\
\text { B/A }\end{array}$ & $\begin{array}{l}\text { Nb actifs } \\
\text { occ. '81 C }\end{array}$ & $\begin{array}{l}\text { Nb actifs } \\
\text { occ. '91 D }\end{array}$ & $\begin{array}{l}\text { Evol. } \\
\text { actifs } \\
\text { occ. D/C }\end{array}$ & $\begin{array}{l}\text { Evol. proportion } \\
\text { artistes (B/D)/ } \\
\text { (A/C) }\end{array}$ \\
\hline Bruxelles & 2099 & 2192 & $\mathbf{1 , 0 4}$ & 616147 & 587281 & 0,95 & $\mathbf{1 , 1 0}$ \\
\hline Anvers & 553 & 702 & $\mathbf{1 , 2 7}$ & 336731 & 340511 & $\mathbf{1 , 0 1}$ & $\mathbf{1 , 2 6}$ \\
\hline Bruges & 68 & 138 & $\mathbf{2 , 0 3}$ & 58550 & 64597 & $\mathbf{1 , 1 0}$ & $\mathbf{1 , 8 4}$ \\
\hline Courtrai & 49 & 109 & $\mathbf{2 , 2 2}$ & 21535 & 58673 & $\mathbf{2 , 7 2}$ & 0,82 \\
\hline Gand & 173 & 368 & $\mathbf{2 , 1 3}$ & 150966 & 156897 & $\mathbf{1 , 0 4}$ & $\mathbf{2 , 0 5}$ \\
\hline Genk & 11 & 31 & $\mathbf{2 , 8 2}$ & 8998 & 26967 & $\mathbf{3 , 0 0}$ & 0,94 \\
\hline Hasselt & 50 & 103 & $\mathbf{2 , 0 6}$ & 33444 & 47420 & $\mathbf{1 , 4 2}$ & $\mathbf{1 , 4 5}$ \\
\hline
\end{tabular}




\begin{tabular}{|l|l|l|l|l|l|l|l|}
\hline Louvain & 83 & 128 & $\mathbf{1 , 5 4}$ & 31602 & 63024 & $\mathbf{1 , 9 9}$ & 0,77 \\
\hline Malines & 62 & 74 & $\mathbf{1 , 1 9}$ & 43617 & 48669 & $\mathbf{1 , 1 2}$ & $\mathbf{1 , 0 7}$ \\
\hline Ostende & 41 & 41 & 1,00 & 30312 & 32249 & $\mathbf{1 , 0 6}$ & 0,94 \\
\hline $\begin{array}{l}\text { Saint- } \\
\text { Nicolas }\end{array}$ & 39 & 45 & $\mathbf{1 , 1 5}$ & 10591 & 32943 & $\mathbf{3 , 1 1}$ & 0,37 \\
\hline Turnhout & 24 & 40 & $\mathbf{1 , 6 7}$ & 25078 & 28659 & $\mathbf{1 , 1 4}$ & $\mathbf{1 , 4 6}$ \\
\hline Charleroi & 173 & 99 & 0,57 & 128499 & 121794 & 0,95 & 0,60 \\
\hline Liège & 246 & 327 & $\mathbf{1 , 3 3}$ & 221529 & 199073 & 0,90 & $\mathbf{1 , 4 8}$ \\
\hline Mons & 65 & 28 & 0,43 & 72875 & 67411 & 0,93 & 0,47 \\
\hline Namur & 62 & 44 & 0,71 & 48945 & 50777 & $\mathbf{1 , 0 4}$ & 0,68 \\
\hline Tournai & 54 & 23 & 0,43 & 31408 & 30971 & 0,99 & 0,43 \\
\hline Verviers & 48 & 51 & $\mathbf{1 , 0 6}$ & 33298 & 32322 & 0,97 & $\mathbf{1 , 0 9}$ \\
\hline Toutes & 3900 & 4543 & $\mathbf{1 , 1 6}$ & 1904125 & 1990238 & $\mathbf{1 , 0 5}$ & $\mathbf{1 , 1 1}$ \\
\hline Belgique & 5117 & 6543 & $\mathbf{1 , 2 8}$ & 3519070 & 3645052 & $\mathbf{1 , 0 4}$ & $\mathbf{1 , 2 3}$ \\
\hline
\end{tabular}

Source : INS - Recensement de la population, 1981 \& 1991

Les deux régions urbaines ayant connu la plus forte croissance de la proportion d'artistes au sein de leur population active occupée entre 1981 et 1991 sont celles de Gand et Bruges, pour lesquelles cette proportion a (presque) doublé. Elles sont suivies dans l'ordre par celles de Liège, Turnhout et Hasselt. Anvers se place juste devant Bruxelles, où la proportion d'artistes a évolué au même rythme que la population active dans son ensemble. Les villes qui ont vu augmenter la présence d'artistes au sein de leur population durant cette période sont principalement des villes flamandes : au contraire, la plupart des villes wallonnes sont en perte de vitesse sur le plan artistique.

Le plus petit rapport, signifiant une nette diminution de la proportion d'artiste, est cependant le fait d'une ville flamande, Saint-Nicolas. L'observation de l'évolution du nombre d'artistes et de celui de la population active occupée permet néanmoins de se rendre compte que ce résultat fait suite à une très forte croissance de cette dernière. Un processus identique est à l'oeuvre, bien que de manière moins saisissante, dans les autres villes flamandes ayant vu leur proportion d'artistes diminuer (Louvain, Courtrai, Ostende et Genk). En Wallonie, la situation de Tournai, Mons, Charleroi et Namur (rapports inférieurs à 0,70 ) procède d'un tout autre phénomène. Dans un contexte de crise économique liée à la désindustrialisation, les trois premières ont vu leur population active occupée diminuer durant la décennie 1981-1991, ainsi que leur population d'artistes (et ce à un rythme plus rapide). Il est intéressant de remarquer que Liège et Bruxelles - ainsi que Verviers - ont également connu une diminution de leur population 
active occupée, mais n'en ont pas moins continué à attirer les artistes. Dans le cas des deux grandes villes, la présence d'institutions prestigieuses, le volume et la diversité des artistes et publics qui y résidaient sont restés des attraits pour les artistes, tandis que les plus petites villes ne bénéficiant pas des mêmes avantages ont vu leur population artistique s'éroder.

Bien qu'imparfait puisque n'étant construit que sur une partie des professions artistiques, le rapport de la proportion d'artistes dans la population active occupée des villes aux deux années analysées permet de mieux comprendre les différences observées entre les deux régions. Entre 1981 et 1991, les régions urbaines flamandes ont vu leur population d'artistes augmenter (et ce à un rythme parfois plus élevé que ne croissait leur population active occupée) : à la fin de la période, ces villes se caractérisent par une proportion d'artistes importante au sein de leur population active occupée totale. Durant la même période, la plupart des régions urbaines de Wallonie ont perdu des artistes : en 1991, elles se distinguent par un nombre d'artistes étonnamment bas par rapport à leur taille et une proportion d'artistes dans la population active occupée plus faible. La capitale enfin, quoiqu'elle ait également souffert de la période de crise des années 1980, n'en est pas moins restée attractive pour les artistes, qui en 1991 s'y concentrent de manière évidente, tant de manière absolue que relative.

La décennie 1980 correspond par ailleurs à l'approfondissement du processus de fédéralisation de l'Etat belge, à la suite des premières réformes instaurant notamment en 1980 la compétence communautaire en matière de culture. Dès ce moment, les politiques culturelles menées au nord et au sud du pays ont pu se différencier, se traduisant par des investissements ou des politiques d'austérité selon le contexte économique et les décisions politiques prises en la matière. Ayant connu une meilleure conjoncture économique durant les années 1980, il semble logique que la Flandre dans son ensemble ait accueilli et accueille au début des années 1990 plus d'artistes que la partie sud du pays, une meilleure situation sur ce plan pouvant se traduire par plus d'investissements publics et privés - dans les infrastructures culturelles et le financement de projets, ainsi que par l'existence d'un public aisé.

Le fait que les évolutions du nombre d'artistes les plus importantes ne correspondent pas aux villes ayant vu croître le plus leur population active occupée montre par ailleurs que d'autres éléments que la seule croissance de la population interviennent pour expliquer la localisation des artistes, qui rendent certains espaces urbains plus attractifs que d'autres pour cette population.

\section{Conclusion}

Envisagés comme des éléments-clés du dynamisme et de la croissance économique dans un contexte concurrentiel croissant entre les espaces urbains, artistes et activités culturelles ont été l'objet de nombreux travaux depuis une vingtaine d'années, principalement en Amérique du Nord. L'intérêt des auteurs - et des autorités locales réside dans l'analyse de la répartition de ce groupe professionnel particulier et la recherche de relations bénéfiques entre le secteur culturel et le développement économique local. Dans le cadre de cet article, c'est au premier aspect que je me suis intéressée : sur base de données du recensement de la population de 1991, la répartition des artistes professionnels en Belgique a été étudiée en envisageant la diversité des 
disciplines qu'ils exercent, l'évolution temporelle de leur présence (décennie 1980) et en variant les échelles d'analyse spatiale (comparaisons inter- et intra-urbaines).

L'utilisation de telles données statistiques, souvent critiquées du fait de leur capacité imparfaite à cerner la population des artistes, permet pourtant l'obtention de résultats intéressants. En premier lieu, une différence prononcée se marque entre les deux grandes régions du pays, la Flandre abritant proportionnellement plus d'artistes que la Wallonie. La faiblesse de la présence des artistes dans les villes wallonnes (hormis Liège) résulte notamment d'une diminution de leur nombre et de la part qu'ils occupent parmi les actifs occupés au cours de la décennie 1980, dans un contexte économique plus défavorable en Wallonie. Ces observations reflètent toutefois une situation passée, avant l'adaptation des villes au nouveau contexte postindustriel; si des écarts existent encore certainement aujourd'hui entre la Flandre et la Wallonie sur le plan artistique, la situation au sud du pays s'est probablement améliorée ces dernières années, ce qu'une actualisation des résultats - rendue compliquée du fait de modifications apportées au recensement pourrait vérifier 9

52 Le deuxième constat confirme ce qui a été observé ailleurs, c'est-à-dire le rôle polarisateur qu'exercent les régions urbaines - et leur partie centrale - sur la population artistique dans son ensemble et particulièrement dans le cas des praticiens des arts de la scène, qui sont moins représentés à mesure que l'on s'éloigne des noyaux urbains.

53 Les deux plus grandes régions urbaines du pays, Bruxelles et Anvers, se distinguent des autres selon le nombre absolu et relatif d'artistes, mais surtout par le fait qu'elles abritent une plus grande variété de professions artistiques, concentrant notamment les artistes créateurs. Anvers apparaît ainsi comme une véritable métropole, qui réussit à attirer les artistes et la création flamande au même titre que la capitale nationale. En Wallonie, Liège est sans conteste la ville qui concentre le plus d'artistes, avec la particularité d'être très spécialisée dans un domaine, la musique. Elle héberge toutefois relativement moins de créateurs (dans le domaine des arts de la scène, par opposition aux artistes interprètes) que les deux plus grandes villes du pays, ce qui permet de penser que c'est à Bruxelles que se concentre la création francophone.

En l'absence de relation évidente entre la taille des villes et le nombre d'artistes ainsi qu'entre la croissance de la population active et celle de la population artistique, d'autres facteurs devront être pris en compte dans la suite de ces recherches pour expliquer la situation observée au niveau des régions urbaines belges. Dans le cas des villes américaines, Blau et al. considèrent par exemple différentes variables explicatives liées aux conditions sociales et économiques des villes, mesurées à travers des indicateurs du statut professionnel, du niveau d'éducation ou de revenus de la population (Blau et al., 1985).

D'autre part, nous avons vu que toutes les formes d'art ne sont pas représentées à part égale dans les 18 régions urbaines; en Wallonie, cela a sans doute influencé la décision de la Communauté française de spécialiser Bruxelles, Liège et le Hainaut respectivement dans le théâtre, la musique et enfin la danse et les arts visuels à l'occasion de l'implantation de nouvelles infrastructures culturelles, telles que le nouveau Théâtre National, Charleroi-Danses ou le MAC's (Vincent \& Wunderle, 2002, p. 12). L'apparition de ces nouvelles structures après 1991 et la disparition d'autres ont probablement contribué à modifier la répartition des artistes entre les régions urbaines. 

artistique pourrait donc constituer un critère explicatif supplémentaire de la géographie des artistes, particulièrement dans le cas des professionnels de la scène qui sont amenés à $\mathrm{y}$ travailler ${ }^{10}$. Le cas de Liège est sans doute exemplatif à ce sujet, la ville hébergeant entre autres deux grandes structures de musique classique, un conservatoire et se particularisant par une forte concentration de musiciens. La présence de ces derniers pourrait toutefois également dépendre de bonnes structures d'enregistrement musical: un grand nombre d'entreprises liées aux secteurs d'activités culturels participent en effet à la diffusion des oeuvres auprès du public tout en offrant des possibilités de travail aux artistes.

La répartition des emplois de ces secteurs d'activités mériterait in fine d'être analysée et comparée à celles des artistes : elle permettrait d'appréhender le poids de ces secteurs dans les différentes régions urbaines du pays et ce faisant, d'approfondir la compréhension des logiques et enjeux liés à la répartition des artistes, tout en rendant possible l'amorce d'une réflexion sur l'impact économique local des activités artistiques, souvent invoqué, toujours discuté mais rarement démontré.

\section{BIBLIOGRAPHIE}

BAINA. L. (2003), “Constructing contemporary artistic identities in Toronto neighbourhoods”, The Canadian Geographer, 47, 3, pp. 303-317.

BECKER H.S. (1982), Les mondes de l'art, Paris, Flammarion.

BLAU J.R., BLAU P.M., GOLDEN R.M. (1985), “Social inequality and the arts”, American Journal of Sociology, 91, 2, pp. 309-331.

COMMISSION EUROPÉENNE (2000), Les statistiques culturelles dans l'UE. Rapport final du LEG, Eurostat Working papers.

FLORIDA R. (2002), The rise of the creative class : and how it's transforming work, leisure, community and everyday life, New York, Basic Books.

GLAESER E. L. (2005), “Review of Richard Florida's The rise of the Creative Class”, Regional Science \& Urban Economics, 35, pp. 593-596.

GLENN L. (2008), “La culture des Capitales ?”, C4, novembre (disponible sur : http://

c4.agora.eu.org/).

HEILBRUN J. (1992), “Art and culture as central place functions”, Urban Studies, 29, pp. 205-215.

HOUBEN C., MASQUELIER J-M. (2009), Cartographie des opérateurs culturels subventionnés en Communauté française, Observatoire des Politiques culturelles, Ministère de la Communauté française (disponible sur Internet : http://www.opc.cfwb.be/index.php?id=3847\#c7610 - dernière consultation : septembre 2009). 
JAUMAIN M. (2002), "Elaboration de statistiques et indicateurs culturels au sein d'un Etat fédéral : le cas de la Belgique", communication au colloque Les statistiques face aux défis de la diversité culturelle dans un contexte de globalisation, Paris-Montréal, octobre 2002.

KARTTUNEN S. (1998), "How to identify artists ? Defining the population for 'status-of-the-artist' studies", Poetics, 26, pp. 1-19.

LEVINE M.V. (2004), La “classe créative" et la prospérité urbaine : mythes et réalités, conférence du 20 mai 2004, INRS-Urbanisation, Culture et Société, Montréal.

LLOYD R. (2002), “Neo-bohemia : art and neighbourhood redevelopment in Chicago", Journal of Urban Affairs, 24, 5, pp. 517-532.

LOOSE M., LAMBERTS M. (2006), Kunst en kunde. Aanzet tot een monitoringinstrument voor de artistieke sector, onderzoek in opdracht van Kunstenloket vzw, Hoger Instituut voor de Arbeid (K.U.Leuven), Leuven.

LUYTEN S., VAN HECKE E. (2007), “De Belgische stadsgewesten 2001”, Statistics Belgium Working Paper, 14, Algemene Directie Statistiek en Economische Informatie, Brussel.

MARKUSEN A. (2006), "Urban development and the politics of a creative class : evidence from a study of artists", Environment and Planning A, 38, pp. 1921-1940.

MARKUSEN A., SCHROCK G. (2006), "The artistic dividend : urban artistic specialization and economic development implications", Urban Studies, 43, 10, pp. 1661-1686.

MARKUSEN A., SCHROCK G., CAMERON M. (2004), The artistic dividend revisited, Project on regional and industrial economics, University of Minnesota.

MARKUSEN A., KING D. (2003), The artistic dividend : the arts' hidden contributions to regional development, Project on regional and industrial economics, University of Minnesota.

MENET B. (1998), “Etat de la danse en Communauté française. Un diagnostic délicat”, Balcon/ Balkon, 2, pp. 83-96.

MILES S., PADDISON R. (2005), "Introduction : The rise and rise of culture-led urban regeneration", Urban Studies, 42, 5/6, pp. 833-839.

MITCHELL C. J. A., BUNTING T., PICCIONI M. (2004), "Visual artists : counter-urbanites in the Canadian countryside”, The Canadian Geographer, 48, 2, pp. 152-167.

MOULIN R. (1983), “De l'artisan au professionnel : l'artiste”, Sociologie du Travail, 4, pp. 388-403. PECK J. (2005), "Struggling with the creative class", International Journal of Urban and Regional Research, 29, 4, pp. 740-770.

SCHIMPF M., SEREDA P. (2007), “Vers une géographie de la culture : l'écart entre l'urbain et le rural en matière de professions culturelles", Culture, tourisme et Centre de la statistique de l'éducation, document de recherche, n 81-595-MIF2007053, Statistique Canada.

SCOTT A.J. (1997), “The cultural economy of cities", International Journal of Urban and Regional Research, 21, 2, pp. 323-339.

SHEARMUR R. (2005), L'aristocratie mobile du savoir : quelques réflexions sur les thèses de Richard Florida, communication présentée au Congrès annuel de l'Association d'économie politique, novembre 2005, Montréal.

VINCENT A., WUNDERLE M. (2002), Les acteurs économiques de la culture, Bruxelles, CRISP .

VIVANT E. (2006), “La classe créative existe-t-elle ?”, Les Annales de la Recherche Urbaine, 101, pp. 155-161. 


\section{NOTES}

1. C'est donc bien des individus exerçant une activité artistique qu'il s'agit et non pas de l'ensemble des personnes travaillant dans les secteurs d'activités culturels (activités annexes de la production et de la diffusion des oeuvres par exemple, qui font partie de ce que Becker nomme les "mondes de l'art" - 1982).

2. Outre le manque de détail dans les classifications, qui génère le regroupement d'items différents dans une même catégorie, celles-ci peuvent être incomplètes : "Le secteur culturel connaît des changements rapides et des nouvelles professions émergent, ou le statut de certaines professions évolue (par exemple, de technique à artistique). C'est la raison pour laquelle les nomenclatures sont toujours datées et ne peuvent pas, suffisamment, prendre en compte la diversité de la vie culturelle. Ce fait doit être accepté, mais il ne doit pas être un obstacle pour un travail statistique sur ce champ" (Commission européenne, 2000, p. 81). Le mauvais état des nomenclatures concernant le secteur artistique s'explique donc par la difficulté de définir un groupe dont les limites sont floues et mouvantes et par le caractère récent de l'intérêt pour ce secteur d'un point de vue statistique.

3. Lorsque l'information est disponible, certains auteurs choisissent de considérer également les personnes dont seule la profession secondaire est artistique. Ce choix n'a pas été fait ici.

4. Ceci d'autant plus que Mons a été désignée capitale culturelle de la Wallonie en 1978 (les titres de capitales politique, sociale et économique ayant été respectivement attribués à Namur, Charleroi et Liège). Le fait que la culture ne soit pas une compétence régionale explique probablement le manque d'investissements pour des projets culturels et la situation observée en 1991. Il est à noter qu'au cours des années 1990, de nouveaux projets ont vu le jour (MAC's, Manège.Mons, etc.) suite à la volonté du gouvernement régional et du gouvernement de la Communauté de positionner la ville comme véritable capitale culturelle wallonne - qui aboutira à la reconnaissance officielle du titre en 2002 (Glenn, 2008).

5. De façon générale, ces communes non-urbaines se caractérisent par des proportions plus faibles d'artistes dans la population. Cependant, un retour à la carte fait ressortir certaines communes périphériques des villes, où les proportions d'artistes ne sont pas négligeables. Outre le fait que les artistes ont pu participer au même titre que le reste de la population à la périurbanisation, ceci peut également traduire la recherche de la nature et d'un environnement tranquille pour travailler, à proximité de la ville et du marché de l'art (cf. périphérie bruxelloise sud). Mitchell et al. ont étudié ce phénomène dans des localités rurales périurbaines au Canada 2004.

6. Une tentative a été faite de séparer les professions du dernier groupe sur base du secteur d'activité économique déclaré lors du recensement, mais elle n'a pas donné de résultats concluants du fait du caractère insatisfaisant de la nomenclature NACE (entre autres, l'existence d'une large catégorie "professions libérales artistiques et littéraires" qui regroupe environ $20 \%$ des artistes recensés en 1991, sans possibilité de différenciation des types de disciplines exercés).

7. Cette constatation se base sur les déclarations de la profession principale : cependant, à l'instar des croisements entre disciplines artistiques, les fonctions exercées par les artistes peuvent être nombreuses. Un musicien se décrivant comme tel peut en réalité également composer, tout comme un danseur peut occasionnellement chorégraphier un spectacle...

8. La spécificité de la danse à Anvers et à Liège rappelle la présence d'écoles importantes. Plusieurs écoles de danse existent à Anvers, notamment le Stedelijk Instituut voor Ballet, le Ballet Royal de Flandre (spécialisé en danse classique) ; la spécificité liégeoise en 1991 procède elle sans doute du Ballet de l'Opéra Royal de Wallonie et de son école de danse classique, tous deux disparus aujourd'hui (Menet, 1998). 
9. Un aperçu - imparfait - de l'évolution ultérieure du secteur artistique dans les régions urbaines peut être obtenu sur base des données de l'ONSS et l'INASTI relatives aux emplois, en considérant l'évolution des secteurs d'activité dans lesquels travaillent le plus souvent les artistes identifiés en 1991 (notamment la catégorie des professions libérales artistiques et littéraires). Si le nombre d'emplois de ces secteurs a effectivement crû en Wallonie durant la décennie 1990, cette croissance s'est toutefois produite à un rythme moins élevé que dans les villes flamandes, ce qui laisse présager une perpétuation du contraste observé sur le plan artistique entre les villes du sud et du nord du pays.

10. Un obstacle important à la réalisation d'un tel relevé réside dans l'attribution des compétences culturelles aux Communautés, dont découle l'absence de données centralisées sur les opérateurs culturels, ainsi que l'a relevé Jaumain dans un texte traitant de la mise en place d'indicateurs et statistiques culturels en Communauté française (2002). Ceci explique qu'à ce jour nous ne disposons pas d'une base de données complète de ces infrastructures.

\section{RÉSUMÉS}

S'insérant dans la problématique des liens entre culture et développement urbain, cette étude présente la répartition spatiale des artistes en Belgique et compare sur ce point les régions urbaines du pays. Souvent évoqué, le rôle des créateurs dans la dynamique économique des villes est généralement envisagé de manière simpliste; des travaux ont pourtant montré que la géographie des artistes est un apport important et pertinent sur lequel une analyse des rapports entre activités culturelles et dynamiques urbaines contemporaines peut être bâtie. L'article esquisse la présence artistique en Belgique, à travers trois dimensions - la prise en compte de plusieurs échelles d'analyse spatiale, la distinction des disciplines artistiques et l'évolution temporelle de la présence des artistes. Celles-ci permettent de mieux appréhender les particularités et différences existant sur ce plan entre les territoires et d'envisager des pistes pour approfondir la compréhension des logiques et enjeux liés à la répartition des artistes, rendant possible l'amorce d'une réflexion sur l'impact économique local des activités artistiques.

This paper presents the spatial distribution of artists in the different urban areas of Belgium, with regard to the issue of links between culture and urban development. The role of creators in cities' economic processes, though often mentioned, is generally considered in simplistic terms. However, studies have shown that the geography of artists is a significant and pertinent contribution upon which an analysis of the relations between cultural activities and contemporary urban dynamics can be built.

Three dimensions are taken into account: several scales of spatial analysis, distinction between artistic disciplines, and temporal evolution of artists' presence. These three dimensions allow us to better understand the features and differences peculiar to the territories and to imagine new paths for a deepened comprehension of the logics and issues related to artists' distribution, making it possible to initiate a reflection on the local economic impact of artistic activities. 
INDEX

Mots-clés : développement urbain, régions urbaines, Belgique

Keywords : artists, urban development, urban areas, Belgium

\section{AUTEUR}

TATIANA DEBROUX

Aspirante F.R.S.-FNRS, Laboratoire de géographie humaine, Université Libre de Bruxelles, tdebroux@ulb.ac.be 